\title{
REVIEW
}

\section{Assessment of vaccination strategies against highly pathogenic avian influenza in China}

\author{
Honglei SUN, Jinhua LIU (ه) \\ Key Laboratory of Zoonosis of Ministry of Agriculture, College of Veterinary Medicine, \\ China Agricultural University, Beijing 100193, China
}

\begin{abstract}
Vaccination for highly pathogenic avian influenza (HPAI) has been implemented in China for a decade, however, the virus is still present in poultry. A series of recombinant vaccines, Re-1 to Re-7, have been developed and used, and Re- 8 will also be used in clinical settings to prevent the prevailing flu strains. The question remains, when can China eradicate the disease? Here, we review the epidemiology of H5 HPAI along with the development, usage and problems of vaccines. Further suggestions for controlling the disease in China are provided.
\end{abstract}

Keywords highly pathogenic avian influenza, vaccine, vaccination, control

\section{Introduction}

China is one of the largest countries for poultry production in the world. However, more than half of the poultry are bred in small-scale farms or in backyards. This type of breeding style enables domestic fowls to share the same niches with wild birds, allowing domestic fowls to be an important intermediate host in the transfer of influenza from wild birds to domestic animals [1], which poses great difficulties for the control of avian influenza in China. In 2004, a combined culling plus vaccination strategy was used to control outbreaks of highly pathogenic avian influenza (HPAI) in China [2]. However, up to 2014, 127 outbreaks in domestic poultry had occurred in over 20 provinces, causing severe economic damage to the poultry industry in China (http://www.oie.int).

\section{Outbreaks of H5 avian influenza and vaccines used in China}

H5N1 avian influenza viruses were first detected in sick

Received November 28, 2014; accepted December 24, 2014

Correspondence: 1jh@cau.edu.cn geese in Guangdong province in 1996 [3]. This is the first documented H5N1 HPAI virus isolation in China. Since 1999, H5N1 viruses have been repeatedly isolated from apparently healthy ducks in southern China [4]. In 2001 and 2003, two H5N1 viruses were isolated from pigs in Fujian Province [5]. On January 27, 2004, a highly pathogenic $\mathrm{H} 5 \mathrm{~N} 1$ virus caused an outbreak in domestic ducks in Guangxi Zhuang Autonomous Region, and then more than 40 outbreaks occurred in 16 provinces within the next month [2]. To control the outbreaks of highly pathogenic avian influenza, an inactivated oil-emulsified vaccine was developed using an $\mathrm{H} 5 \mathrm{~N} 2$ low pathogenic virus, $\mathrm{A} /$ Turky/England/N-28/73, as a seed virus [2]. The H5N2 vaccines played an important role in the rapid control of the H5N1 outbreaks in China in 2004. However, the $\mathrm{H} 5 \mathrm{~N} 2$ vaccine seed virus exhibited antigenic diversity with the prevalent H5N1 strains in China. To solve the problem, Re-1, a reassortant virus, was created through plasmid based reverse genetics, with the haemagglutinin (HA) and neuraminidase (NA) genes from the A/goose/ Guangdong/1/1996 (GS/GD/1/96) virus and the internal genes from the high growth A/Puerto Rico/8/34 (PR8) virus [6]. The vaccine was approved to be used in the field by the end of 2004 .

In 2006, there were 10 outbreaks on small-scale, backyard chicken or duck farms, with birds that had not been vaccinated. It is important to note that $\mathrm{H} 5 \mathrm{~N} 1$ outbreaks occurred in some layer farms in Shanxi Province, although the chickens in these farms were vaccinated with the $\mathrm{H} 5$ inactivated vaccines. Samples were sent to the National Avian Influenza Reference Laboratory for disease diagnosis. An $\mathrm{H} 5 \mathrm{~N} 1$ influenza virus, A/ chicken/Shanxi/2/06 (CK/SX/06) was identified, but this strain reacted poorly with the antisera against Re-1 virusbased antigen. Sequence analysis indicated that this H5N1 virus was also genetically quite different from the $\mathrm{H} 5 \mathrm{~N} 1$ viruses isolated from other locations [7]. To control the epidemics caused by the CK/SX/06 virus, a new inactivated vaccine $\mathrm{Re}-4$ was developed by using a recombinant $\mathrm{H} 5 \mathrm{~N} 1 / \mathrm{PR} 8$ reassortant virus bearing the 
modified HA and NA genes from the CK/SX/06 virus. The vaccine was applied in the selected provinces in northern China in 2006. In 2006, Smith et al. reported the emergence and predominance in poultry in China since late 2005 of a previously uncharacterized $\mathrm{H} 5 \mathrm{~N} 1$ virus sublineage (Fujian-like) [8]. Viruses from this sublineage gradually replaced those multiple regional distinct sublineages and caused human infection in China. The virus was transmitted to Hong Kong, Laos, Malaysia, and Thailand, resulting in a new wave of transmission and outbreaks in South-east Asia. In May 2008, another new H5N1/PR8 reassortant virus, Re-5, which derives its HA and NA genes from A/duck/Anhui/1/06, was applied in China.

The lack of uniformity in nomenclature often leads to confusion in the interpretation of the evolutionary relationships among HPAI virus (H5N1) isolates. To address the issue, the World Health Organization/World Organisation for Animal Health/Food and Agriculture Organization H5N1 Evolution Working Group developed a unified nomenclature system for the classification of H5N1 HPAI viruses and 10 distinct clades of viruses (0-9) were initially designated to characterize the phylodynamics of the HA gene of the Gs/GD-like H5 viruses [9]. After 2008, molecular epidemiological surveys indicated that commercial farms have been well protected by the vaccines, but the $\mathrm{H} 5 \mathrm{~N} 1$ viruses are still circulating in China, especially in live bird markets, and backyard flocks are at greater risk of infections $[7,10]$. Phylogenetic analysis indicated that three clades $(2.3 .4,2.3 .2$ and 7$)$ co-circulated in poultry in China in 2007-2009 [10]. The variant of clade 2.3.2 was first isolated in Hong Kong SAR and mainland China in 2007 $[11,12]$. It has since circulated widely in poultry in China and caused a new wave of cross-continental infection, spreading from Asia to Europe [13-15]. Antigenic analysis revealed that the clade 2.3.2 viruses reacted weakly with antisera prepared from the Re-4 and Re-5 vaccines widely used in China [10]. The new H5N1/PR8 reassortant virus Re-6 was developed and applied in China from 2011.

After 2007, clade 7.2 viruses were predominantly found in chickens in Northern China. Although the viruses from this clade were genetically similar to the Re-4 virus, they reacted poorly with the antisera raised against Re- 4 virusbased antigen [10]. In December 2013, there was an outbreak of H5N2 HPAI in a chicken farm in Hebei Province. Phylogenetic analysis revealed that the virus was a reassortant of the $\mathrm{H} 5 \mathrm{~N} 1$ and $\mathrm{H} 9 \mathrm{~N} 2$ viruses [16]. To control the epidemics, Re-7 vaccine was approved for use in China. In addition to H5N1 HPAI viruses, the emergence of novel H5N2, H5N5, H5N6, and H5N8 subtype HPAI viruses adds more evidence for the active evolution of H5 viruses in China [17-20]. These novel H5 viruses reacted poorly with antisera prepared from the Re-5 vaccine, although they belong to the same clade. To control these novel H5 viruses, a new vaccine Re- 8 was developed. This new vaccine is expected to be approved for use in the future. The present facts indicate that although vaccination has been implemented in China for the past ten years, $\mathrm{H} 5$ avian influenza still continues to be found in poultry populations.

Table 1 shows $\mathrm{H} 5$ avian influenza outbreaks and vaccines used in China from 2004 to 2014.

\section{Problems in implementing the vaccination strategy}

\subsection{Farm management}

Clinical data have shown that vaccination was not ideal and had some drawbacks. Most of the influenza vaccines could prevent clinical signs but not infection [21-23]. The

Table 1 H5 avian influenza outbreaks and vaccines used in China from 2004 to 2014

\begin{tabular}{lccc}
\hline \multirow{2}{*}{ Year } & Outbreaks & \multicolumn{2}{c}{ Vaccine } \\
\cline { 2 - 4 } 2004 & 50 & Subtype & A/turky/England/N-28/73 \\
2005 & 32 & H5N2 & H5N1(A/goose/Guangdong/1/1996)/PR8 (Re-1) \\
2006 & 12 & - & - \\
2007 & 4 & H5N1 & H5N1(A/chicken/Shanxi/2/2006)/PR8 (Re-4) \\
2008 & 6 & H5N1 & H5N1(A/duck/Anhui/1/2006)/PR8 (Re-5) \\
2009 & 2 & - & - \\
2010 & 0 & - & H5N1(A/duck/Guangdong/S1322/2010)/PR8 (Re-6) \\
2011 & 1 & H5N1 & - \\
2012 & 8 & - & H5N1(A/Chicken/Liaoning/S4092/2011)/PR8 (Re-7) \\
2013 & 2 & - & - \\
2014 & 9 & H5N1 & \\
total & 126 & - & \\
\hline
\end{tabular}


culling plus vaccination policy for preventing HPAI H5N1 viruses was implemented in China in 2004 [2], requiring all poultry to be vaccinated. However, there is still a large proportion of poultry raised in small-scale, free-range contexts in China. Shoddy rearing equipment, poor prevention conditions and extensive management are the main problems for such poultry production. Furthermore, it is not easy to carry out an effective vaccination program for these farms. All these deficiencies resulted in ineffective immunization in these poultry. As a consequence of smallscale, free-range poultry, it is difficult for the government to supervise and provide epidemic prevention services. Therefore, free-range poultry are often missed in programs for preventing and controlling avian influenza epidemics, and therefore represent a disease risk.

\subsection{Quality of the vaccine}

The government has required $100 \%$ vaccine coverage in domestic poultry, and financial support from the government ensures the implementation of this strategy. However, many farmers lack the enthusiasm to use the vaccine. They complain of low quality vaccines containing suboptimal concentration of antigens that are passed the quality guarantee dates. In addition, inactivated vaccines induce an antibody peak at least 14 days after immunization [24]. The short growing period of meat poultry and vaccine residues in the tissue of immunized poultry restrict its comprehensive usage in poultry meat production.

\subsection{Antigenic diversity}

The H5N1 HPAI viruses in the natural environment are not a homogeneous population, and they may have different antigenicity $[7,10]$. Thus, after exposure to the field viruses, vaccinated birds become infected without signs of disease but excrete small amount of the viruses from the respiratory tract and/or in the feces [10,21-23]. This phenomenon may also cause some new problems for the control of the disease. First, infections with the HPAI viruses in vaccinated birds may cause silent spread of the virus between flocks when biosecurity is weak [25]. Secondly, antibody pressure may drive the viral evolution $[26,27]$. Influenza A virus is an RNA virus which has an polymerase enzyme lacks proofreading activity, resulting in a replication mistake in every $10^{-4}$ nucleotides per se [28]. Populations of viruses in a host then appear as quasispecies. The quasi-species which are the most fit in a particular environment would become the predominant population. Thus, new viral strains would have a selective advantage for spreading in vaccinated flocks, if they have become antigenically different from the vaccine strains [26].

In addition, many low level biosecurity farms ignore effective management strategies and readily use vaccine to prevent diseases. Controlling the infection source, cutting off the route of transmission, and protecting susceptible populations are three prevention strategies to control the infectious disease. When biosecurity is compromised, poultry are often exposed to viruses in the field. In this situation, vaccination alone will not prevent the spread of the viruses because the transmission rate of HPAI viruses is very high $[25,29]$.

\section{Suggestions for controlling avian influenza}

\subsection{Improving traditional poultry breeding practices}

Good farm management and strict biosecurity measures help prevent virus entry into farms and transmission between sheds within farms [30]. A retrospective analysis showed that most of the H5N1 outbreaks in China occurred on small-scale or backyard farms [2]. Some small-scale farms showed a low immunity level. Also, free-range domestic fowls have potential contact with wild waterfowls and other domestic animals such as pigs. Therefore, these farms are important in transmission of virus from wild birds to other domestic poultry and other animals, including humans [1,31]. Thus, the traditional poultry raising practices in China must be improved to control H5N1 HPAI. A poultry farm should only raise one kind of poultry, and avoid mixed production of chickens with either ducks or geese. We strongly suggest that the government set up an "admission threshold" for approval to breed poultry.

\subsection{Stopping live poultry markets}

Epidemiology surveillance has shown that the live poultry markets have an important role in the amplification and dissemination of influenza viruses [32,33], and the exposure history in live poultry markets is the key risk factor for human $\mathrm{H} 5 \mathrm{~N} 1$ and $\mathrm{H} 7 \mathrm{~N} 9$ cases [34-36]. In the 1997 Hong Kong influenza incident and the 2013 H7N9 human infection in Shanghai, stopping the live poultry markets proved to be a very useful measure to control human infection. Therefore, the following measures are suggested: slaughtering the poultry in specialized factories with refrigeration, and eventually sending the fresh-kept poultry product to supermarkets for sale. The strategy may not only break the transmission route of the virus, but also prevent human contact with live poultry, thereby stopping infection of people.

\subsection{Improving the prevention system for animal epidemics}

An effective animal epidemic prevention and control system is the best option to control avian influenza [30]. 
However, China's animal epidemic prevention system does not satisfy the requirements yet. Although mechanism for emergency reactions are ready for the prevention and control of major animal diseases such as the avian influenza, and planning has been undertaken, the relatively poor system in rural areas makes it impossible to meet the demand of higher standard of prevention and control of avian influenza. Problems have appeared in some districts where the suspected avian influenza could not be diagnosed in time, which eliminated the best opportunity for prevention. It is essential to find and eradicate outbreaks promptly to control HPAI [30]. In addition, the government should establish regional avian influenza laboratories in each province in addition to the National Avian Influenza Reference Laboratory. The China Animal Health and Epidemiology Center could then manage the prevalence and distribution of avian influenza based on annual epidemiological surveys, and consequently perform risk assessment and guide prevention work.

Furthermore, the government should also put in place comprehensive mechanisms of culling compensation. Once culling measures are performed, full compensation should be immediately provided to the farmers so they do not suffer a financial loss. Only in this way they will report an outbreak quickly instead of concealing the truth, and this will help the government to take prompt measures to control outbreaks of HPAI.

\section{Conclusions}

China has employed a culling plus vaccination strategy to control H5N1 avian influenza for a decade. The use of influenza vaccine increased the resistance of poultry to the disease and played an important role in controling the transmission of avian influenza. However, clinical data have shown some problems during implementation of the vaccination strategy. Avian influenza has been the biggest barrier in the development of the poultry industry. Therefore, great efforts should be made to change avian production practices, develop standardized and large-scale poultry farms, change poultry consumption habits, and improve the animal epidemic prevention and control systems. With these problems resolved, vaccination programs can be stopped, and monitoring and culling policies adopted, as carried out in developed countries, and avian influenza ultimately controlled.

Acknowledgements This work was supported by the National Basic Research Program (2011CB504702) and the National Science Fund for Distinguished Young Scholars (31025029).

Compliance with ethics guidelines Honglei Sun and Jinhua Liu declare that they have no conflict of interest or financial conflicts to disclose.

This article does not contain any studies with human or animal subjects performed by any of the authors.

\section{References}

1. Hulse-Post D J, Sturm-Ramirez K M, Humberd J, Seiler P, Govorkova E A, Krauss S, Scholtissek C, Puthavathana P, Buranathai C, Nguyen T D, Long H T, Naipospos T S P, Chen H, Ellis T M, Guan Y, Peiris J S M, Webster R G. Role of domestic ducks in the propagation and biological evolution of highly pathogenic H5N1 influenza viruses in Asia. Proceedings of the National Academy of Sciences of the United States of America, 2005, 102(30): 10682-10687

2. Chen H L. H5N1 avian influenza in China. Science in China Series C: Life Sciences, 2009, 52(5): 419-427

3. Xu X, Subbarao K, Cox N J, Guo Y. Genetic characterization of the pathogenic influenza A/Goose/Guangdong/1/96 (H5N1) virus: similarity of its hemagglutinin gene to those of $\mathrm{H} 5 \mathrm{~N} 1$ viruses from the 1997 outbreaks in Hong Kong. Virology, 1999, 261(1): 1519

4. Chen H, Deng G, Li Z, Tian G, Li Y, Jiao P, Zhang L, Liu Z, Webster R G, Yu K. The evolution of H5N1 influenza viruses in ducks in southern China. Proceedings of the National Academy of Sciences of the United States of America, 2004, 101(28): 1045210457

5. Zhu Q, Yang H, Chen W, Cao W, Zhong G, Jiao P, Deng G, Yu K, Yang C, Bu Z, Kawaoka Y, Chen H. A naturally occurring deletion in its NS gene contributes to the attenuation of an $\mathrm{H} 5 \mathrm{~N} 1$ swine influenza virus in chickens. Journal of Virology, 2008, 82(1): 220228

6. Tian G, Zhang S, Li Y, Bu Z, Liu P, Zhou J, Li C, Shi J, Yu K, Chen H. Protective efficacy in chickens, geese and ducks of an H5N1inactivated vaccine developed by reverse genetics. Virology, 2005, 341(1): 153-162

7. Li Y, Shi J, Zhong G, Deng G, Tian G, Ge J, Zeng X, Song J, Zhao D, Liu L, Jiang Y, Guan Y, Bu Z, Chen H. Continued evolution of H5N1 influenza viruses in wild birds, domestic poultry, and humans in China from 2004 to 2009. Journal of Virology, 2010, 84(17): 8389-8397

8. Smith G J D, Fan X H, Wang J, Li K S, Qin K, Zhang J X, Vijaykrishna D, Cheung C L, Huang K, Rayner J M, Peiris J S M, Chen H, Webster R G, Guan Y. Emergence and predominance of an H5N1 influenza variant in China. Proceedings of the National Academy of Sciences of the United States of America, 2006, 103 (45): 16936-16941

9. WHO OIE. Toward a unified nomenclature system for highly pathogenic avian influenza virus (H5N1). Emerging Infectious Diseases, 2008, 14(7): e1

10. Jiang W M, Liu S, Chen J, Hou G Y, Li J P, Cao Y F, Zhuang Q Y, Li Y, Huang B X, Chen J M. Molecular epidemiological surveys of H5 subtype highly pathogenic avian influenza viruses in poultry in China during 2007-2009. Journal of General Virology, 2010, 91 (10): 2491-2496

11. Smith G J D, Vijaykrishna D, Ellis T M, Dyrting K C, Leung Y H C, Bahl J, Wong C W, Kai H, Chow M K W, Duan L, Chan A S L, Zhang L J, Chen H, Luk G S M, Peiris J S M, Guan Y. Characterization of avian influenza viruses A (H5N1) from wild 
birds, Hong Kong, 2004-2008. Emerging Infectious Diseases, 2009, 15(3): 402-407

12. Chen J, Fang F, Yang Z, Liu X, Zhang H, Zhang Z, Zhang X, Chen Z. Characterization of highly pathogenic $\mathrm{H} 5 \mathrm{~N} 1$ avian influenza viruses isolated from poultry markets in central China. Virus Research, 2009, 146(1-2): 19-28

13. Boltz D A, Douangngeun B, Phommachanh P, Sinthasak S, Mondry R, Obert C, Seiler P, Keating R, Suzuki Y, Hiramatsu H, Govorkova E A, Webster R G. Emergence of H5N1 avian influenza viruses with reduced sensitivity to neuraminidase inhibitors and novel reassortants in Lao People's Democratic Republic. Journal of General Virology, 2010, 91(4): 949-959

14. Nguyen T, Davis C T, Stembridge W, Shu B, Balish A, Inui K, Do H T, Ngo H T, Wan X F, McCarron M, Lindstrom S E, Cox N J, Nguyen C V, Klimov A I, Donis R O. Characterization of a highly pathogenic avian influenza $\mathrm{H} 5 \mathrm{~N} 1$ virus sublineage in poultry seized at ports of entry into Vietnam. Virology, 2009, 387(2): 250-256

15. Uchida Y, Mase M, Yoneda K, Kimura A, Obara T, Kumagai S, Saito T, Yamamoto Y, Nakamura K, Tsukamoto K, Yamaguchi S. Highly pathogenic avian influenza virus (H5N1) isolated from whooper swans, Japan. Emerging Infectious Diseases, 2008, 14(9): 1427-1429

16. Ci Y. Wang D, Fan Z, Liu L, Jia Y, Li X, Tian G, Zeng X, Chen H, $\mathrm{Li}$ Y. Biological analysis and pathogenicity of an $\mathrm{H} 5 \mathrm{~N} 2$ avian influenzavirus isolated from chicken. Chinese Journal of Preventive Veterinary Medicine, 2014, 36(9): 672-675 (in Chinese)

17. Zhao G, Gu X, Lu X, Pan J, Duan Z, Zhao K, Gu M, Liu Q, He L, Chen J, Ge S, Wang Y, Chen S, Wang X, Peng D, Wan H, Liu X. Novel reassortant highly pathogenic H5N2 avian influenza viruses in poultry in China. PLoS ONE, 2012, 7(9): e46183

18. Gu M, Liu W, Cao Y, Peng D, Wang X, Wan H, Zhao G, Xu Q, Zhang W, Song Q, Li Y, Liu X. Novel reassortant highly pathogenic avian influenza (H5N5) viruses in domestic ducks, China. Emerging Infectious Diseases, 2011, 17(6): 1060-1063

19. Zhao K, Gu M, Zhong L, Duan Z, Zhang Y, Zhu Y, Zhao G, Zhao M, Chen Z, Hu S, Liu W, Liu X, Peng D, Liu X. Characterization of three $\mathrm{H} 5 \mathrm{~N} 5$ and one H5N8 highly pathogenic avian influenza viruses in China. Veterinary Microbiology, 2013, 163(3): 351-357

20. Qi X, Cui L, Yu H.Whole-genome sequence of a reassortant H5N6 avian influenza virus isolated from a live poultry market in China, 2013. Genome Announcements, 2014, 2(5): e00706-14

21. Swayne D E, Beck J R, Perdue M L, Beard C W. Efficacy of vaccines in chickens against highly pathogenic Hong Kong H5N1 avian influenza. Avian Diseases, 2001, 45(2): 355-365

22. Liu M, Wood J M, Ellis T, Krauss S, Seiler P, Johnson C, Hoffmann E, Humberd J, Hulse D, Zhang Y, Webster R G, Perez D R. Preparation of a standardized, efficacious agricultural $\mathrm{H} 5 \mathrm{~N} 3$ vaccine by reverse genetics. Virology, 2003, 314(2): 580-590

23. Qiao C, Yu K, Jiang Y, Jia Y, Tian G, Liu M, Deng G, Wang X, Meng Q, Tang X. Protection of chickens against highly lethal H5N1 and $\mathrm{H} 7 \mathrm{~N} 1$ avian influenza viruses with a recombinant fowlpox virus co-expressing H5 haemagglutinin and N1 neuraminidase genes. Avian Pathology, 2003, 32(1): 25-31

24. Qiao C, Tian G, Jiang Y, Li Y, Shi J, Yu K, Chen H. Vaccines developed for H5 highly pathogenic avian influenza in China. Annals of the New York Academy of Sciences, 2006, 1081(1): 182192

25. Savill N J, St Rose S G, Keeling M J, Woolhouse M E J. Silent spread of H5N1 in vaccinated poultry. Nature, 2006, 442(7104): 757

26. Lee C W, Senne D A, Suarez D L. Effect of vaccine use in the evolution of Mexican lineage H5N2 avian influenza virus. Journal of Virology, 2004, 78(15): 8372-8381

27. Peng Y, Zou Y, Li H, Li K, Jiang T. Inferring the antigenic epitopes for highly pathogenic avian influenza H5N1 viruses. Vaccine, 2014, 32(6): 671-676

28. Grenfell B T, Pybus O G, Gog J R. Unifying the epidemiological and evolutionary dynamics of pathogens. Science, 2004, 303(5656): 327-332

29. van der Goot J A, Koch G, De Jong M C M, van Boven M. Quantification of the effect of vaccination on transmission of avian influenza (H7N7) in chickens. Proceedings of the National Academy of Sciences of the United States of America, 2005, 102(50): 1814118146

30. Capua I, Marangon S. Control of avian influenza in poultry. Emerging Infectious Diseases, 2006, 12(9): 1319-1324

31. Beigel J H, Farrar J, Han A M. Avian influenza A (H5N1) infection in humans. New England Journal of Medicine, 2005, 353(13): 1374-1385

32. Kung N Y, Morris R S, Perkins N R, Sims L D, Ellis T M, Bissett L, Chow M, Shortridge K F, Guan Y, Peiris M J S. Risk for infection with highly pathogenic influenza A virus (H5N1) in chickens, Hong Kong, 2002. Emerging Infectious Diseases, 2007, 13(3): 412-418

33. Santhia K, Ramy A, Jayaningsih P, Samaan G, Putra A A G, Dibia N, Sulaimin C, Joni G, Leung C Y H, Malik Peiris J S, Wandra T, Kandun N. Avian influenza A H5N1 infections in Bali Province, Indonesia: a behavioral, virological and seroepidemiological study. Influenza and Other Respiratory Viruses, 2009, 3(3): 81-89

34. Shortridge K F, Gao P, Guan Y, Ito T, Kawaoka Y, Markwell D, Takada A, Webster R G. Interspecies transmission of influenza viruses: H5N1 virus and a Hong Kong SAR perspective. Veterinary Microbiology, 2000, 74(1-2): 141-147

35. Mounts A W, Kwong H, Izurieta H S, Ho Y, Au T, Lee M, Buxton Bridges C, Williams S W, Mak K H, Katz J M, Thompson W W, Cox N J, Fukuda K. Case-control study of risk factors for avian influenza A (H5N1) disease, Hong Kong, 1997. Journal of Infectious Diseases, 1999, 180(2): 505-508

36. Ai J, Huang Y, Xu K. Case-control study of risk factors for human infection with influenza A (H7N9) virus in Jiangsu Province, China, 2013. Euro Surveillance: European Communicable Disease Bulletin, 2013, 18(26): 20510 